Y.-K. Lau

Nagoya Math. J.

Vol. 165 (2002), 71-78

\title{
ON A GENERALIZED DIVISOR PROBLEM I
}

\author{
YUK-KAM LAU
}

\begin{abstract}
We give a discussion on the properties of $\Delta_{a}(x)(-1<a<0)$, which is a generalization of the error term $\Delta(x)$ in the Dirichlet divisor problem. In particular, we study its oscillatory nature and investigate the gaps between its sign-changes for $-1 / 2 \leq a<0$.
\end{abstract}

\section{$\S 1$. Introduction}

Let $\sigma_{a}(n)=\sum_{d \mid n} d^{a}$ and define for $-1<a<0$ and $x \geq 1$,

$$
\Delta_{a}(x)=\sum_{n \leq x}^{\prime} \sigma_{a}(n)-\zeta(1-a) x-\frac{\zeta(1+a)}{1+a} x^{1+a}+\frac{1}{2} \zeta(-a)
$$

where the last term in the sum is halved when $x$ is an integer. The limit function $\lim _{a \rightarrow 0-} \Delta_{a}(x)$ is the same as the classical error term $\Delta(x)$. The determination of its precise order of magnitude, called the Dirichlet's divisor problem, remains open to date. On the other hand, the highly oscillatory behaviour of $\Delta(x)$ has attracted the attention of many authors. There are numerous papers devoted to the study of properties of $\Delta(x)$, such as its power moments, $\Omega_{ \pm}$-results, gaps between sign-changes and etc.. Correspondingly not many results are known for $\Delta_{a}(x)$. The mean square result is a mature one among them. In 1995, Meurman [9] proved that

$$
\int_{2}^{T} \Delta_{a}(x)^{2} d x= \begin{cases}c_{1} T^{3 / 2+a}+O(T) & \text { for }-1 / 2<a<0 \\ c_{2} T \log T+O(T) & \text { for } a=-1 / 2 \\ O(T) & \text { for }-1<a<-1 / 2\end{cases}
$$

where $c_{1}=(6+4 a)^{-1} \pi^{-2} \zeta(3 / 2-a) \zeta(3 / 2+a) \zeta(3 / 2)^{2} \zeta(3)^{-1}, \quad c_{2}=$ $\zeta(3 / 2)^{2} /(24 \zeta(3))$ and the constants implied by the $O$-symbols may depend on $a$. He established the result by using a weighted Voronoi type formula

Received February 8, 2000.

Revised September 22, 2000.

2000 Mathematics Subject Classification: 11N64. 
with an explicit error term of his own. The $O$-terms in the first and the third case are the best possible. The first one was confirmed by Lam and Tsang [7] while, in fact, there exists an asymptotic formula for the third case $(-1<a<-1 / 2)$ in an old paper of Chowla [1] where it is proved that

$$
\int_{2}^{T} \Delta_{a}(x)^{2} d x=c_{3} T+O\left(T^{3 / 2+a} \log T\right)
$$

with $c_{3}=\zeta(-2 a) \zeta(1-a)^{2} /(12 \zeta(2-2 a))$. These mean square results are definitely important. From them, one can see that $a=-1 / 2$ is a 'critical' point for the behaviour of $\Delta_{a}(x)$, for example, the 'average' order of $\Delta_{a}(x)$ is $O\left(x^{1 / 4+a / 2}\right)$ for $-1 / 2<a<0, O(\sqrt{\log x})$ for $a=-1 / 2$ and $O(1)$ for $-1<a<-1 / 2$. Analogous to the case $\Delta(x)$, we expect that $\Delta_{a}(x) \ll$ $x^{1 / 4+a / 2+\epsilon}(-1 / 2 \leq a<0)$ and $\Delta_{a}(x) \ll x^{\epsilon}(-1<a \leq-1 / 2)$, both of which are still open. In the opposite direction, we can find the following $\Omega_{ \pm}$-results: for $-1 / 2 \leq a<0$,

$$
\Delta_{a}(x)=\Omega_{ \pm}\left(x^{1 / 4+a / 2} \log ^{1 / 4+|a| / 2} x\right),
$$

and for $-1<a<-25 / 38$,

$$
\Delta_{a}(x)=\Omega_{ \pm}\left(\exp \left((1+o(1)) \frac{1}{1-|a|}\left(\frac{|a|}{2}\right)^{1-|a|} \frac{(\log x)^{1-|a|}}{\log \log x}\right)\right)
$$

due to Hafner [2] and Pétermann [10] respectively.

As mentioned, the behaviour of $\Delta_{a}(x)$ changes at $a=-1 / 2$. It seems that $\Delta_{a}(x)$ behaves like $\Delta(x)$ only for the range $-1 / 2<a<0$ (or $-1 / 2 \leq$ $a<0)$. We shall investigate the properties of $\Delta_{a}(x)$ at different values of $a$.

In this paper, we shall consider the case $-1 / 2 \leq a<0$ (and the other case in the sequel paper [8]). Our first result is about the difference $\Delta_{a}(x+$ $h)-\Delta_{a}(x)$ for $-1 / 2 \leq a<0$ in the mean. In [5], Jutila proved that for $T^{\epsilon} \ll h \leq \sqrt{T} / 2$,

$$
\int_{T}^{2 T}(\Delta(t+h)-\Delta(t))^{2} d t \asymp T h \log ^{3}(\sqrt{T} / h) .
$$

Results of this kind can reveal their oscillatory natures. Parallel to this, we prove the following: 
THEOREM 1. Let $T \geq 2$ and suppose $a \in[-1 / 2,-\delta]$ where $\delta$ is an arbitrarily small positive number. Then, for $1 \ll h \leq \sqrt{T}$, we have

$$
\int_{T}^{2 T}\left(\Delta_{a}(t+h)-\Delta_{a}(t)\right)^{2} d t \ll_{\delta} T h^{1+2 a} \min \left(\frac{1}{1 / 2-|a|}, \log h\right)
$$

where the implied constant depends only on $\delta$.

Remark. A recent paper of Kiuchi and Tanigawa [6] includes the result of the case $-1 / 2<a<0$ here, but not $a=-1 / 2$.

An application of Theorem 1 is to yield the width of gaps between sign-changes of $\Delta_{a}(x)$.

TheOREM 2. For $-1 / 2 \leq a<0$, we can find a sequence $\left\{T_{n}\right\}$ tending to infinity such that $\Delta_{a}(x)$ has no sign-changes in the interval $\left[T_{n}, T_{n}+c_{a} H_{n}\right]$ where $c_{a}$ is a constant depending only on $a, H_{n}=$ $T_{n}^{1 / 2-|a|(1 / 2+|a|)}\left(\log T_{n}\right)^{-1}$ if $-1 / 2<a<0$ and $H_{n}=\left(\log T_{n}\right)^{3 / 2} /\left(\log \log T_{n}\right)^{2}$ if $a=-1 / 2$.

Concerning the sign-changes of $\Delta_{a}(x)$, by taking $g(n)=\pi^{a / 2} \sigma_{a}(n)$, $\mu(n)=\pi n, r=1+a$ and $\alpha=1$ in Ivić [4, Theorem 1], it follows immediately an upper bound result for the length of gaps between sign-changes.

THEOREM 3. Let $-1 / 2 \leq a \leq 0$ and $T$ be any sufficiently large number. Then, $\Delta_{a}(x)$ has a sign-change in $\left[T, T+c_{a} \sqrt{T}\right]$ for some constant $c_{a}$ depending on a only.

For the case $a=0$, Heath-Brown and Tsang [3] showed in the opposite direction that one can find a sequence $\left\{T_{n}\right\}$ which tends to infinity and $\Delta(x)$ has no sign-changes in the interval $\left[T_{n}, T_{n}+c^{\prime} \sqrt{T_{n}} / \log ^{5} T_{n}\right]$ for some constant $c^{\prime}$. These almost determined the exact order of magnitude of the gaps between sign-changes of $\Delta(x)$. However results of opposite direction for other cases are not known yet. Our Theorem 2, based on the method of Heath-Brown and Tsang, is to furnish this part. It can be seen that the value of $H_{n}$ in Theorem 2 deviates away from $\sqrt{T_{n}}$ as $a$ decreases from 0 to $-1 / 2$. The true order of magnitude of the gaps between sign-changes is still mysterious for such cases. At present, there are not enough information to predict the right order of magnitude.

Finally we want to mention that the $\Omega_{ \pm}$-result in (1.3) for the case $a=-1 / 2$ can be improved. 
Theorem 4. We have

$$
\Delta_{-1 / 2}(x)=\Omega_{ \pm}\left(\exp \left((1+o(1)) \frac{(\log x)^{1 / 2}}{\log \log x}\right)\right) .
$$

It comes from [8, Theorem 1] with $a=-1 / 2$. However, one should note that the definition of $\Delta_{a}(x)$ in [8] is different from here. Let us extend the definition of $\sigma_{a}(n)$ by defining $\sigma_{a}(x)=0$ when $x$ is not a positive integer. Then [8, Theorem 1] gives

$$
\Delta_{-1 / 2}(x)+\frac{1}{2} \sigma_{-1 / 2}(x)=\Omega_{ \pm}\left(\exp \left((1+o(1)) \frac{(\log x)^{1 / 2}}{\log \log x}\right)\right) .
$$

From (1.1), it is apparent that

$$
\Delta_{-1 / 2}(x) \leq \Delta_{-1 / 2}(x)+\frac{1}{2} \sigma_{-1 / 2}(x) \leq \Delta_{-1 / 2}(x+1)+O(1)
$$

and hence Theorem 4 follows. It should be remarked that unlike the case of $-1 / 2 \leq a \leq 0$, Theorem 1 in [8] is derived by another tool instead of the Voronoi-type formula (thus, so is Theorem 4); nonetheless, the method used there in the discussion of sign-changes cannot yield results for the case $a=-1 / 2$. (Note that the results in Theorems 2 and 3 here include this case.) These altogether perhaps give a further support of the peculiarity of $\Delta_{-1 / 2}(x)$.

\section{§2. Proof of Theorem 1}

From [9, Lemma 1] with $X=2 T, Z=4 T$, we have for $t \in[T, 3 T]$,

$$
\Delta_{a}(t)=\Delta_{a}(t, T)+R_{a}(t, T)+O\left(T^{-1 / 4+a / 2}\right)
$$

where

$$
\Delta_{a}(t, T)=\frac{1}{\pi \sqrt{2}} t^{1 / 4+a / 2} \sum_{n \leq 4 T} \frac{\sigma_{a}(n)}{n^{3 / 4+a / 2}} w_{T}(n) \cos \left(4 \pi \sqrt{n t}-\frac{\pi}{4}\right)
$$

and

$$
R_{a}(t, T)=\frac{1}{2 \pi} \sum_{n \leq 4 T} \sigma_{a}(n) \int_{1}^{2} \int_{2 u T}^{\infty} v^{-1} \sin (4 \pi(\sqrt{t}-\sqrt{n}) \sqrt{v}) d v d u
$$


with $w_{T}(u)=1$ for $1 \leq u \leq 2 T$ and $w_{T}(u)=2-u /(2 T)$ for $2 T \leq u \leq 4 T$. Then,

$$
\int_{T}^{2 T}\left(\Delta_{a}(t+h)-\Delta_{a}(t)\right)^{2} d t \ll T+\int_{T}^{2 T}\left(\Delta_{a}(t+h, T)-\Delta_{a}(t, T)\right)^{2} d t
$$

where the mean square value of $R_{a}(\cdot, T)$ is estimated by $[9,(2.3)]$. Now,

$$
\begin{gathered}
\int_{T}^{2 T}\left(\Delta_{a}(t+h, T)-\Delta_{a}(t, T)\right)^{2} d t \\
\ll \int_{T}^{2 T} t^{1 / 2+a}\left|\sum_{n \leq 4 T} \frac{\sigma_{a}(n)}{n^{3 / 4+a / 2}} w_{T}(n)(e(2 \sqrt{n(t+h)})-e(2 \sqrt{n t}))\right|^{2} d t \\
+\int_{T}^{2 T}\left((t+h)^{1 / 4+a / 2}-t^{1 / 4+a / 2}\right)^{2} \\
\times\left|\sum_{n \leq 4 T} \frac{\sigma_{a}(n)}{n^{3 / 4+a / 2}} w_{T}(n) e(2 \sqrt{n(t+h)})\right|^{2} d t
\end{gathered}
$$

$(2.2)=I_{1}+I_{2}$, say.

We split $I_{1}$ into three parts as follows.

$$
\begin{aligned}
& I_{1} \ll \\
& \quad T^{1 / 2+a} \int_{T}^{2 T}\left|\sum_{n \leq T /\left(2 h^{2}\right)} \frac{\sigma_{a}(n)}{n^{3 / 4+a / 2}}(e(2 \sqrt{n}(\sqrt{t+h}-\sqrt{t}))-1) e(2 \sqrt{n t})\right|^{2} d t \\
& \quad+T^{1 / 2+a} \int_{T}^{2 T}\left|\sum_{T /\left(2 h^{2}\right)<n \leq 4 T} \frac{\sigma_{a}(n)}{n^{3 / 4+a / 2}} w_{T}(n) e(2 \sqrt{n(t+h)})\right|^{2} d t \\
& \quad+T^{1 / 2+a} \int_{T}^{2 T}\left|\sum_{T /\left(2 h^{2}\right)<n \leq 4 T} \frac{\sigma_{a}(n)}{n^{3 / 4+a / 2}} w_{T}(n) e(2 \sqrt{n t})\right|^{2} d t \\
& (2.3)=I_{11}+I_{12}+I_{13} .
\end{aligned}
$$

Following the arguments for the estimate of $J^{ \pm}$in $[9$, p.354-355], we see that $I_{2} \ll T$ and $I_{12}, I_{13} \ll T h^{1+2 a} \min \left((1 / 2-|a|)^{-1}, \log h\right)+T$. With $e(2 \sqrt{n}(\sqrt{t+h}-\sqrt{t}))-1 \ll h \sqrt{n / T}$, Second Mean Value Theorem for integrals, we get for some $\xi \in[T, 2 T]$,

$$
I_{11} \ll T^{1 / 2+a} h^{2} \sum_{n \leq T /\left(2 h^{2}\right)} \frac{\sigma_{a}(n)^{2}}{n^{1 / 2+a}}+T^{1+a}\left|\int_{\xi}^{2 T} \sum(t) e(2(\sqrt{m}-\sqrt{n}) \sqrt{t}) \frac{d t}{\sqrt{t}}\right|
$$


where

$$
\begin{aligned}
\sum(t)=\sum_{m \neq n \leq T /\left(2 h^{2}\right)} \frac{\sigma_{a}(m) \sigma_{a}(n)}{(m n)^{3 / 4+a / 2}}(e(2 \sqrt{m}(\sqrt{t+h}-\sqrt{t}))-1) \\
\times \frac{(e(2 \sqrt{n}(\sqrt{t+h}-\sqrt{t}))-1) .}{} .
\end{aligned}
$$

The first summand in the right-hand side is $\ll T h^{1+2 a}$. By integration by parts, we see that the second summand is $\ll T h^{2 a}$ and so $I_{11} \ll T h^{1+2 a}$. Our theorem follows from (2.1)-(2.3).

Remark. A careful treatment, following the same line of arguments in Jutila [5], can furthermore lead to $\int_{T}^{2 T}\left(\Delta_{a}(t+h)-\Delta_{a}(t)\right)^{2} d t \gg_{\delta} T h^{1+2 a}$. This was not done here for simplicity.

\section{§3. Proof of Theorem 2}

Following the method in [3], we first show that for $1 \ll H \leq \sqrt{T}$,

$$
\begin{aligned}
& \int_{T}^{2 T} \max _{h \leq H}\left(\Delta_{a}(t+h)-\Delta_{a}(t)\right)^{2} d t \\
\ll & T\left(H \log H \min \left(\frac{1}{1 / 2-|a|}, \log H\right)\right)^{1 /(1+|a|) .}
\end{aligned}
$$

To prove it, let us write $H=2^{\lambda} b, \lambda \in \mathbf{N}$. Since for $v \leq u$,

$$
\begin{aligned}
& \Delta_{a}(u)-\Delta_{a}(v)= \\
& \sum_{v<n \leq u}{ }^{\prime} \sigma_{a}(n)-\zeta(1-a)(u-v)-\frac{\zeta(1+a)}{1+a}\left(u^{1+a}-v^{1+a}\right) \\
& \geq-O(|u-v|),
\end{aligned}
$$

we have for $j b<h \leq(j+1) b$,

$$
\begin{aligned}
\Delta_{a}(t+j b)-\Delta_{a}(t)-O(b) & \leq \Delta_{a}(t+h)-\Delta_{a}(t) \\
& \leq \Delta_{a}(t+(j+1) b)-\Delta_{a}(t)+O(b) .
\end{aligned}
$$

Hence, for a fixed $t$, let $\left|\Delta_{a}(t+h)-\Delta_{a}(t)\right|$ attain a maximum at $h_{0}=h_{0}(t)$ over $[0, H]$, we then have

$$
\begin{aligned}
\max _{h \leq H}\left|\Delta_{a}(t+h)-\Delta_{a}(t)\right| & =\left|\Delta_{a}\left(t+h_{0}\right)-\Delta_{a}(t)\right| \\
& \leq \max _{1 \leq j \leq 2^{\lambda}}\left|\Delta_{a}(t+j b)-\Delta_{a}(t)\right|+O(b) .
\end{aligned}
$$


Now, let $\max _{1 \leq j \leq 2^{\lambda}}\left|\Delta_{a}(t+j b)-\Delta_{a}(t)\right|=\left|\Delta_{a}\left(t+j_{0} b\right)-\Delta_{a}(t)\right|$ for some $j_{0}=j_{0}(t)$, by writing $j_{0}=2^{\lambda} \sum_{\mu} 2^{-\mu}$ where the sum runs over a certain set $S_{t}$ of non-negative integers $\mu \leq \lambda$, we can express it as

$$
\Delta_{a}\left(t+j_{0} b\right)-\Delta_{a}(t)=\sum_{\mu}\left(\Delta_{a}\left(t+(\nu+1) 2^{\lambda-\mu} b\right)-\Delta_{a}\left(t+\nu 2^{\lambda-\mu} b\right)\right)
$$

where $0 \leq \nu=\nu_{t, \mu}<2^{\mu}$ is an integer. (To be specific, $\nu=2^{\mu} \sum_{\alpha} 2^{-\alpha}$ where $\alpha$ runs over $S_{t}$ and satisfies $\min S_{t} \leq \alpha<\mu$.) By Cauchy-Schwarz's inequality, we have

$$
\begin{aligned}
& \left(\Delta_{a}\left(t+j_{0} b\right)-\Delta_{a}(t)\right)^{2} \\
\leq & \left(\sum_{\mu} 1\right)\left(\sum_{\mu}\left(\Delta_{a}\left(t+(\nu+1) 2^{\lambda-\mu} b\right)-\Delta_{a}\left(t+\nu 2^{\lambda-\mu} b\right)\right)^{2}\right) \\
\leq & (\lambda+1) \sum_{\mu} \sum_{0 \leq \nu<2^{\mu}}\left(\Delta_{a}\left(t+(\nu+1) 2^{\lambda-\mu} b\right)-\Delta_{a}\left(t+\nu 2^{\lambda-\mu} b\right)\right)^{2},
\end{aligned}
$$

after including all other integers $\nu \in\left[0,2^{\mu}\right)$. Thus, by taking $b=$ $\left(H \log H \min \left((1 / 2-|a|)^{-1}, \log H\right)\right)^{1 /(2+2|a|)}$, we obtain with Theorem 1 ,

$$
\begin{aligned}
& \int_{T}^{2 T} \max _{h \leq H}\left(\Delta_{a}(t+h)-\Delta_{a}(t)\right)^{2} d t \\
\ll & \lambda \sum_{\mu \leq \lambda} \sum_{0 \leq \nu<2^{\mu}} \int_{T+\nu 2^{\lambda-\mu} b}^{2 T+\nu 2^{\lambda-\mu} b}\left(\Delta_{a}\left(t+2^{\lambda-\mu} b\right)-\Delta_{a}(t)\right)^{2} d t+T b^{2} \\
\ll & T\left(H \log H \min \left(\frac{1}{|a|-1 / 2}, \log H\right)\right)^{1 /(1+|a|)} .
\end{aligned}
$$

Applying (3.1) with $H=c_{a} T^{(1 / 2+a)(1+|a|)} / \log T$ for $-1 / 2<a<0$ and $H=c_{a}(\log T)^{3 / 2} /(\log \log T)^{2}$ for $a=-1 / 2$ for some suitable small constant $c_{a}>0$, we see together with (1.2) that the integral

$$
\int_{T}^{2 T}\left(\Delta_{a}(t)^{2}-\max _{h \leq H}\left(\Delta_{a}(t+h)-\Delta_{a}(t)\right)^{2}\right) d t
$$

is positive. Our assertion then follows.

\section{REFERENCES}

[1] S. Chowla, Contributions to the analytic theory of numbers, Math. Z., 35 (1932), 279-299. 
[2] J.L. Hafner, On the Average Order of a Class of Arithmetical Functions, J. Number Theory, 15 (1982), 36-76.

[3] D.R. Heath-Brown and K. Tsang, Sign Changes of $E(T), \Delta(x)$ and $P(x)$, J. Number Theory, 49 (1984), 73-83.

[4] A. Ivić, Large values of certain number-theoretic error terms, Acta Arith., 56 (1990), 135-159.

[5] M. Jutila, On the divisor problem for short intervals, Ann. Univ. Turkuensis Ser. A, I 186 (1984), 23-30.

[6] I. Kiuchi and Y. Tanigawa, The mean value theorem of the divisor problem for short intervals, Arch. Math. (Basel), 71 (1998), 445-453.

[7] K.-Y. Lam and K.-M. Tsang, The Mean Square of the Error Term in a Generalization of the Dirichlet Divisor Problem, Analytic Number Theory, edited by Y.Motohashi, Cambridge University Press, 1997.

[8] Y.-K. Lau, On a generalized divisor problem II, manuscript.

[9] T. Meurman, The mean square of the error term in a generalization of Dirichlet's divisor problem, Acta Arith., 74 (1996), 351-361.

[10] Y.-F.S. Pétermann, About a Theorem of Paolo Codecà's and Omega Estimates for Arithmetical Convolutions, Second Part, Ann. Scuola Norm. Sup. Pisa Cl. Sci. (4), 17 (1990), 343-353.

Institut Élie Cartan

Université Henri Poincaré (Nancy 1)

54506 Vandoeuvre lés Nancy Cedex, France

CURRENT ADDRESS

Department of Mathematics

The University of Hong Kong

Pokfulam Road, HONG KONG

yklau@maths.hku.hk 